\title{
Damage localization in plate-like structure using built-in PZT sensor network
}

\author{
Xinglong Liu*1, Chengxu Zhou ${ }^{2}$ and Zhongwei Jiang ${ }^{2}$ \\ ${ }^{1}$ National Engineering Laboratory for System Integration of High Speed Train (South), \\ CSR Qingdao Sifang, Co., Ltd, Qingdao, China \\ ${ }^{2}$ Department of Mechanical Engineering, Engineering Faculty, Yamaguchi University, Ube, Japan
}

(Received May 8, 2010, Revised January 20, 2011, Accepted November 8, 2011)

\begin{abstract}
In this study, a Lamb-wave based damage detection approach is proposed for damage localization in plate. A sensor network consisting of three PZT wafer type actuators/sensors is used to generate and detect Lamb waves. To minimize the complication resulted from the multimode and dispersive characteristics of Lamb waves, the fundamental symmetric Lamb mode, $S_{0}$ is selectively generated through designing the excitation frequency of the narrowband input signal. A damage localization algorithm based upon the configuration of the PZT sensor network is developed. Time-frequency analysis method is applied to purify the raw signal and extract damage features. Experimental result obtained from aluminum plate verified the proposed damage localization approach.
\end{abstract}

Keywords: damage localizations; structural health monitoring; Lamb waves; piezoelectric sensors; timefrequency analysis.

\section{Introduction}

Lamb waves, guided elastic wave in plate-like structure, have shown promising potential for structural health monitoring (SHM) due to its high capability to detect and characterize both surface and internal damage in structures and ability of interrogating large areas with a small number of transducers. The advances in sensor and hardware technology have led to extensive study on the use of Lamb waves for SHM with integrated piezoelectric sensors (Giurgiutiu et al. 2002, Wieslaw et al. 2009, Lin and Yuan 2002, Grondel et al. 2002).

In the past few years many Lamb wave-based SHM techniques have been developed to identify and locate damage in large plate using distributed piezoelectric sensor network. Tua et al. (2004) proposed the elliptical loci method for crack localization and evaluation. Four piezoelectric actuators/sensors placed on a square grid configuration are used to excite and sense Lamb waves. Several elliptical loci are constructed based upon the time of flights (TOFs) of crack-reflected Lamb waves. However, the major drawback of this method is the existence of blind zones. Su et al. (2002) utilized a distributed sensor network of four PZT actuators/sensors for damage localization in plate-like structure. Four sets of nonlinear equations are established with time lags via the actuatorsensor paths, and then optimization method is employed to solve the nonlinear equations. Nevertheless,

*Corresponding author, Dr., E-mail: xinglong-liu@hotmail.com 
solving the nonlinear equations is not computationally efficient. Wang and Chang (2001) developed an active diagnostic system to locate damage in composite and concrete materials with four surfacebonded PZT sensors. Based on the TOFs of wave scattered from the damage, an optimization algorithm was utilized to locate the damage. But the TOFs are obtained by subtracting the baseline signal recorded for the undamaged state from the signal recorded for the damage state. Wang and Yuan (2007) developed an active damage localization technique to locate damage in isotropic plate based on the energy propagation of Lamb waves. The damage localization problem is formulated as least-square problem to minimize an error function between the model and the measured data. The sensor network consists of one PZT actuator and three/four PZT sensors. The scattered wave is extracted by subtracting the baseline signal from the signal of damaged plate. Hu el al. (2008) proposed a damage localization algorithm based upon the TOFs of reflection wave from damage, a removable piezoelectric transducer sets consisting of a center actuator and three surrounding sensors are designed to selectively generate $A_{0}$ Lamb wave mode. The advantage of this algorithm is that the baseline signals are not needed.

In this paper, a new damage localization approach with a built-in PZT sensor network consisting of three PZT actuators/sensors is proposed. The fundamental $S_{0}$ Lamb waves mode is selectively generated with PZT actuators/sensors. Signal processing technique is adopted for extracting the TOFs from the acquired Lamb wave signals of damaged plate.

\section{Lamb wave theory}

Lamb waves are a type of elastic perturbation propagating in a solid plate with free-traction boundaries on their both surfaces (Viktorov 1967). This type of wave phenomenon is first described in theory by Horace in 1917. Lamb waves can be classified into two groups based upon the wave motion: symmetric (S) modes and anti-symmetric (A) modes. The Rayleigh-Lamb frequency equation for the symmetric and anti-symmetric modes in an isotropic plate is given by the Eqs. (1)(2) (Achenbach 1984), respectively.

$$
\begin{aligned}
& \frac{\tan \beta d}{\tan \alpha d}=-\frac{\left(\xi^{2}-\beta^{2}\right)^{2}}{4 \alpha \beta \xi^{2}} \\
& \frac{\tan \beta d}{\tan \alpha d}=-\frac{4 \alpha \beta \xi^{2}}{\left(\xi^{2}-\beta^{2}\right)^{2}}
\end{aligned}
$$

where $\xi=2 \pi / \lambda$ is the wave number, and $\alpha^{2}=\omega^{2} / c_{L}^{2}-\xi^{2}, \beta^{2}=\omega^{2} / c_{T}^{2}-\xi^{2} \cdot c_{L}=(\lambda+2 \mu) / \rho$ and $c_{T}=\mu / \rho$ are the longitudinal and shear wave speed, respectively. $\rho$ is density of the plate, $\lambda=E v /((1-$ $2 v)(1+v))$ and $\mu=E /(2(1+v)), E$ is the Young's modulus, and $v$ is Poisson's ratio. The group velocity of the symmetric and anti-symmetric modes is given as

$$
c_{g}=d \omega / d \xi
$$

The Rayleigh-Lamb equations can be solved numerically for a given material, leading to the so-called dispersion characteristics. Fig. 1 displays the phase and group velocity dispersion curves of Lamb waves in an aluminum plate. The dispersion curves show that at low frequency-thickness product, two fundamental modes, $S_{0}$ and $A_{0}$ can propagate, as the frequency-thickness increases, more modes are 


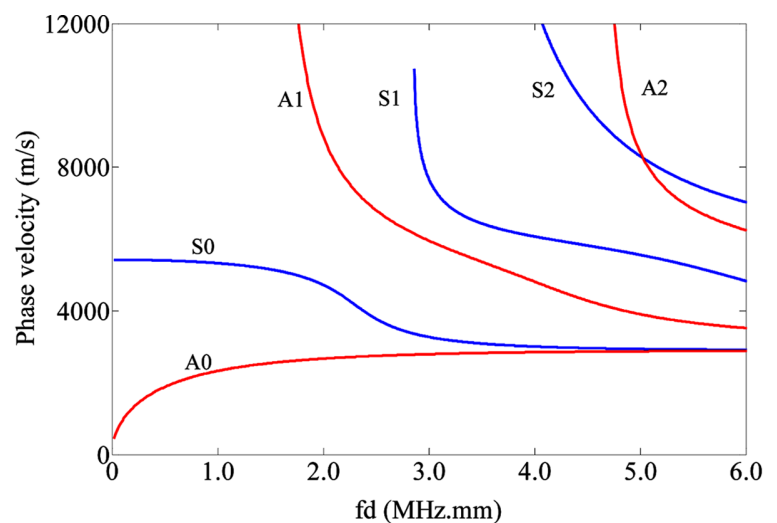

(a)

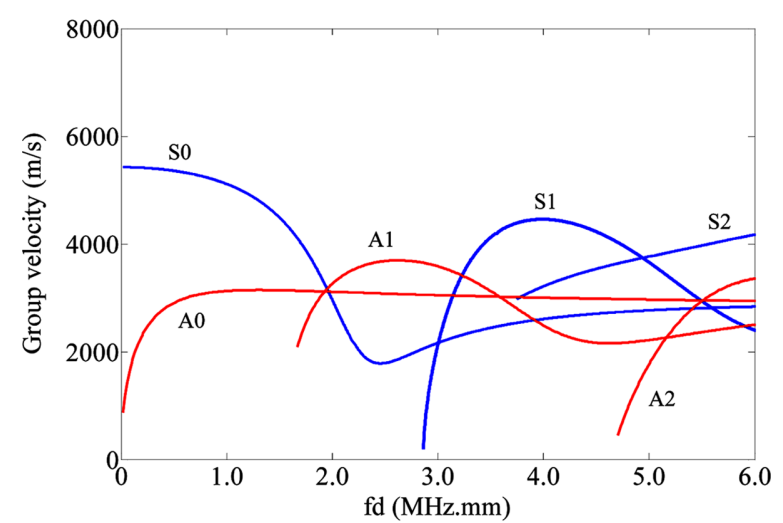

(b)

Fig. 1 Dispersion curves of Lamb waves: (a) phase velocity and (b) group velocity

possible and the interpretation of signals tends to become more complicated. Furthermore, all of the modes are dispersive, that is to say, the shape of pulse signal changes with the propagation time and distance, making the measurement of their arrival times difficult.

The multiple-mode and dispersive characteristics of Lamb waves complicate signal interpretation for damage identification. To alleviate the complication, selective generation and detection of a single mode within a frequency range to minimize the dispersion effect should be adopted. In general, fundamental $A_{0}$ and $S_{0}$ modes are commonly used for damage detection. $S_{0}$ mode is faster and has much lower attenuation than $A_{0}$ modes and hence can propagate longer distance. On the other hand, although $A_{0}$ mode is more sensitive to small damage because of its shorter wavelength, it shows more severe dispersion at low frequencies compared to $S_{0}$ mode. Therefore, $S_{0}$ mode is chosen for damage detection herein.

\section{Selective generation of $S_{0}$ Lamb wave mode}

\subsection{Experimental setups}

The overall test configuration of this study is shown in Fig. 2. The test setup consists of an aluminum plate with surface-bonded PZT actuator/sensors, function generator (Hioki 7078), signal amplifier (HAS 4101NF Corp.) and digital oscilloscope (Yokogama DL 1540C). The dimensions of the plate are $1200 \times 1200 \times 2 \mathrm{~mm}^{3}$. Three circular PZT patches are bonded onto one surface of the plate. The circular PZT actuator/sensor is of $10 \mathrm{~mm}$ in diameter and $0.2 \mathrm{~mm}$ in thickness. The three PZT sensors are placed 120 degrees apart from each other to form a circle with diameter of 100 $\mathrm{mm}$ around the circumference. The center of the circle coincides with the middle position of the plate surface. The configuration of the collocated sensor network is schematically illustrated in the Fig. 2. The damage localization algorithm based upon the configuration of the PZT sensor network will be described in the next section. For this collocated PZT sensor network, the three PZT sensors are alternately served as actuator and sensor to generate and detect Lamb waves. The input signal is a Hanning-windowed tone burst signal, which has narrow bandwidth to concentrate the input energy 


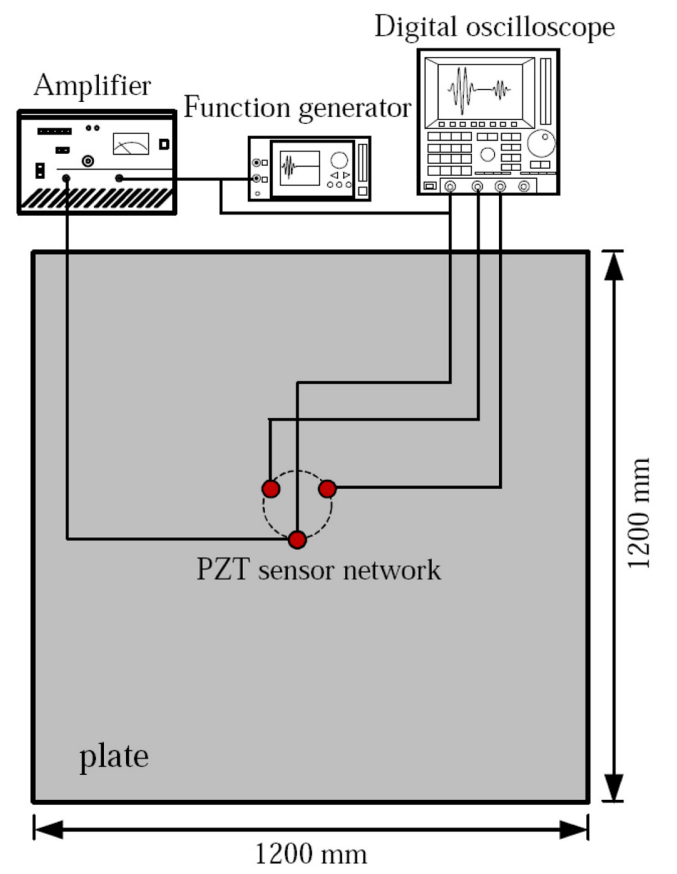

Fig. 2 Schematic of experimental set up
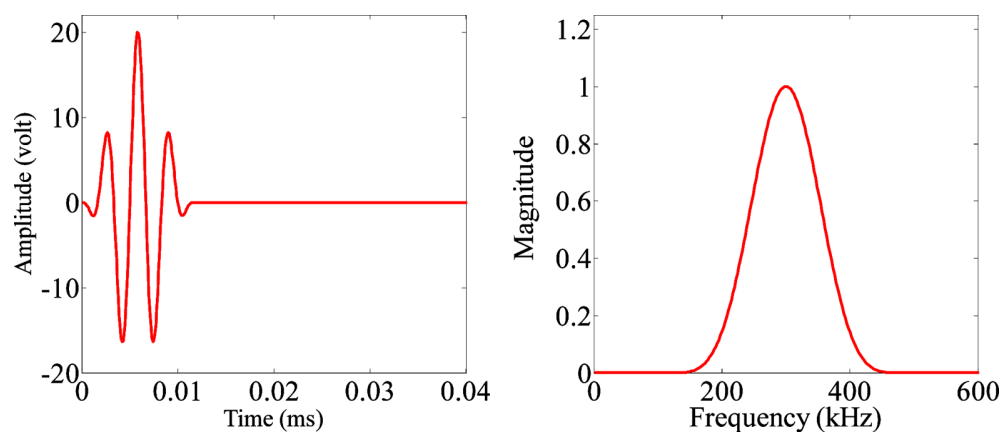

Fig. 3 Tone burst signal and its spectrum

at a central frequency, and hence can alleviate the dispersion of Lamb waves. As an example, a 3.5 counts tone burst signal for the central excitation frequency of $300 \mathrm{kHz}$ is displayed in Fig. 3. The tone burst signal is produced with the function generator at the repetition rate of $100 \mathrm{~Hz}$. The peak voltage of the input signal applied to the PZT actuator/sensor is $20 \mathrm{~V}$. the sampling rate of the digital oscilloscope is set as $10 \mathrm{MHz}$. the Lamb wave response signals recorded by PZT sensors are displayed and stored in the digital oscilloscope.

\subsection{Design of the excitation frequency}

The important procedure for selective generation of a single Lamb wave mode is to design the central excitation frequency of the tone burst signal based upon the dispersion curves. In this study, first the experimental group velocity of $S_{0}$ mode is measured over the frequency range of 75-350 


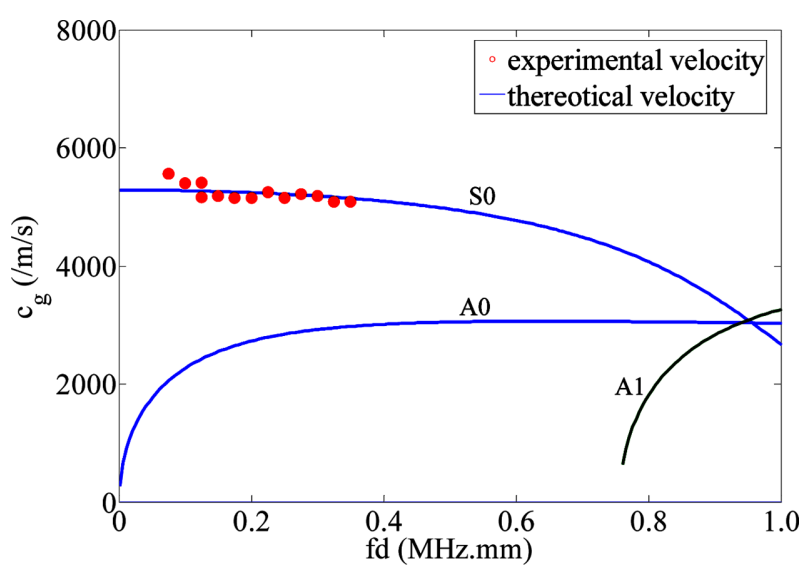

Fig. 4 Comparison of theoretical and experimental wave velocities

Table 1 Material properties of aluminum

\begin{tabular}{ccc}
\hline \hline$E / \mathrm{GP}$ & $\mathrm{v}$ & $\rho / \mathrm{kg} / \mathrm{m}^{3}$ \\
\hline 66.45 & 0.345 & 2700 \\
\hline
\end{tabular}

$\mathrm{kHz}$ in step of $25 \mathrm{kHz}$. For a given set of material properties, the corresponding theoretical group velocities are numerically computed with Eq. (3). Then a least squares method is adopted for choosing such a set of the material properties that the corresponding theoretical group velocity curve matches well with the experimental group velocities. The result of least squares curve-fitting method is shown in Fig. 4 and the determined material properties of the structure are described in Table 1.

The computed dispersion curve is then used as a guideline to design the excitation frequency. First the excitation frequency of the narrowband tone burst signal should be low enough where only $A_{0} / S_{0}$ mode can propagate. At the same time, the excitation frequency ought to be located at a position where the slope of the group velocity curve of $S_{0}$ mode is relatively flat over the frequency band of the excitation signal. Moreover, the wavelength of $S_{0}$ mode at the excitation frequency ought to be sufficiently small so that strong interaction of Lamb waves with damage will occur. After the excitation frequency range is initially determined, then the relation of the wavelengthfrequency is used to choose the excitation frequency at which only a single $S_{0}$ mode is generated. The study done by Giurgiutiu (2005) showed that when the diameter of PZT actuator is equal to odd integer multiple of half wavelength of the particular Lamb mode, strong voltage response for the corresponding Lamb mode will be produced, whereas when the diameter of PZT actuator is equal to even integer multiple of half wavelength of Lamb modes, very weak voltage response will be generated. According to the material properties given in Table 1, the wavelength against the excitation frequency for $A_{0}$ and $S_{0}$ mode is plotted in Fig. 5. From the figure it is observed that at $50 \mathrm{kHz}$ the wavelength of $A_{0}$ mode is about $20 \mathrm{~mm}$, which is nearly twice of the diameter of PZT actuator, and the wavelength of $S_{0}$ mode is over $100 \mathrm{~mm}$. therefore, large voltage amplitude of $A_{0}$ mode will be generated at $50 \mathrm{kHz}$. On the contrary, at $300 \mathrm{kHz}$ the wavelength of $A_{0}$ mode is about $6.5 \mathrm{~mm}$ and that of $S_{0}$ mode is about $17.5 \mathrm{~mm}$. thus only $\mathrm{S}_{0}$ mode can be dominantly generated.

To validate the design procedure for the excitation frequency, the Lamb wave signals for a few excitation frequencies are measured, and several typical signals are shown in Fig. 6. It is seen that $A_{0}$ Lamb wave mode dominate the voltage response at low frequency around $50 \mathrm{kHz}$, whereas $S_{0}$ 


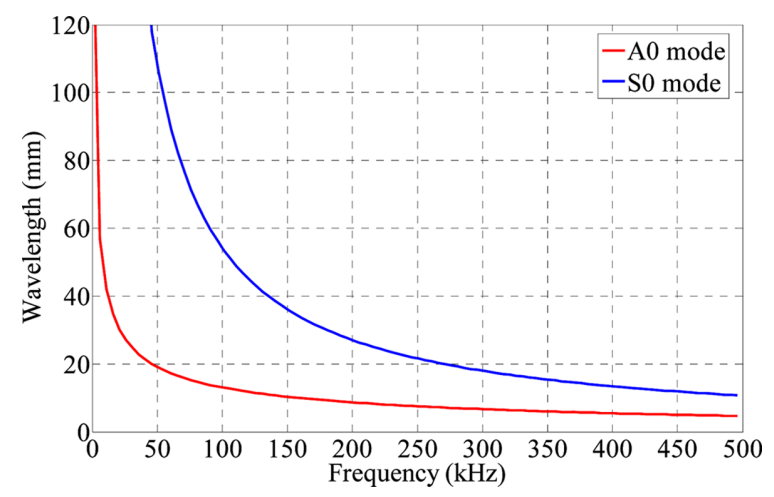

Fig. 5 The wavelength of $A_{0}$ and $S_{0}$ mode versus frequency

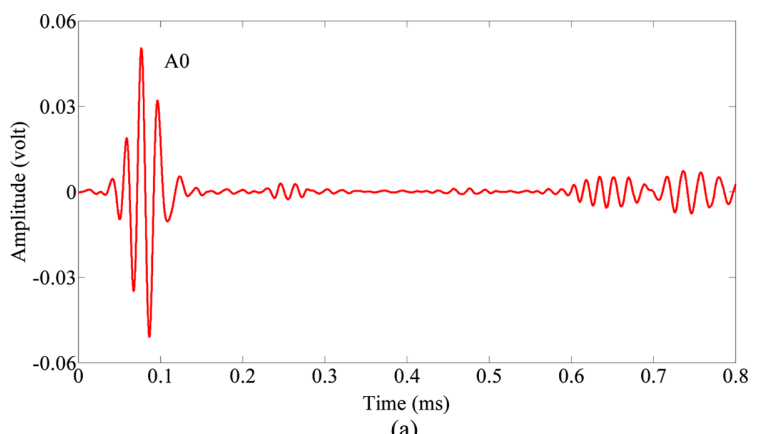

(a)

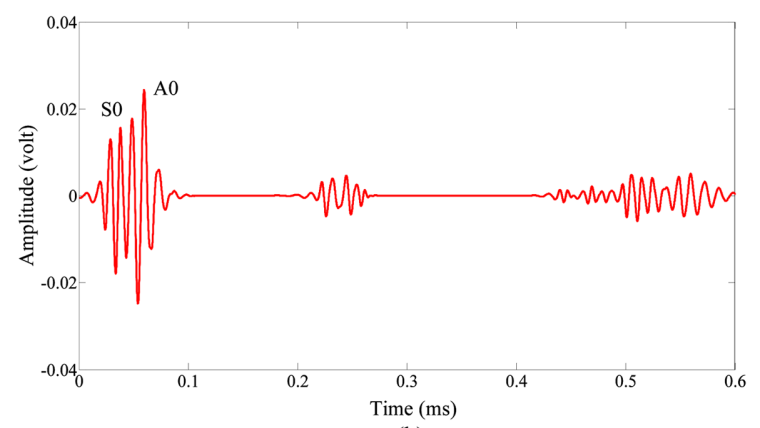

(b)

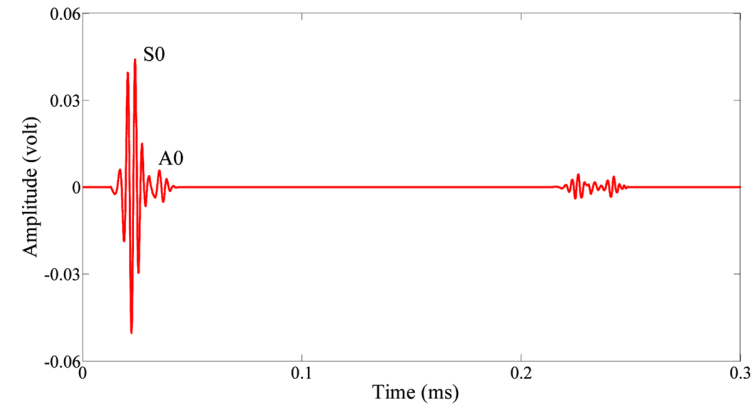

(c)

Fig. $6 \mathrm{Lamb}$ wave signals for the excitation frequency of (a) $50 \mathrm{kHz}$ (b) $100 \mathrm{kHz}$ and (c)300 kHz

Lamb wave mode dominates the voltage response at $300 \mathrm{kHz}$. In order for mainly generating $S_{0}$ Lamb wave mode, the excitation frequency is chosen as $300 \mathrm{kHz}$ in the study.

\section{Algorithm and signal processing}

\subsection{Damage localization algorithm}

The damage localization algorithm based upon the collocated PZT sensor network is proposed in this section. As illustrated in Fig. 7, the placement positions of three circular PZT actuators/sensors are 


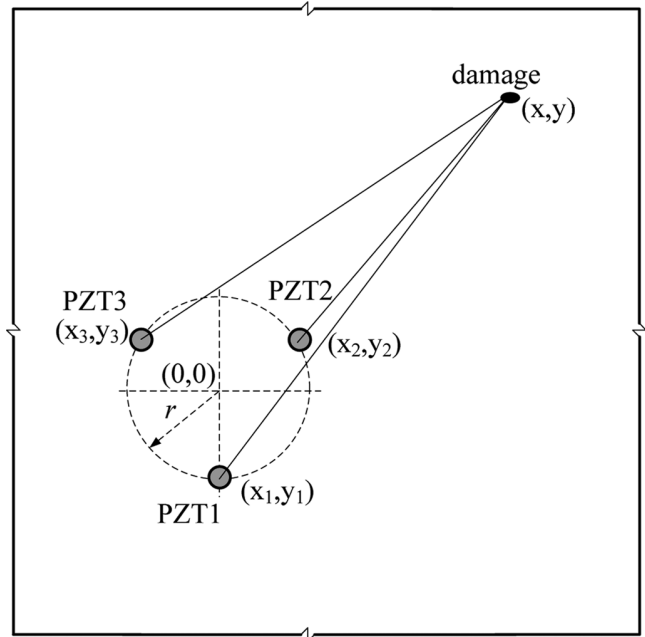

Fig. 7 Illustration of localization algorithm

uniformly located at the circumference of a circle with radius $r$. A Cartesian coordinate system of the sensor-network is used. The origin of the coordinate system coincides with the center of the circle, and $\left(x_{1}, y_{1}\right),\left(x_{2}, y_{2}\right),\left(x_{3}, y_{3}\right)$ are the coordinates of each PZT actuator/sensor's center, respectively. Assume the damage is located at $(x, y)$, the relation between the position of damage and sensors could be described with the following equation

$$
\sqrt{\left(x-x_{i}\right)^{2}+\left(y-y_{i}\right)}=t_{i} c_{g}(i=1,2,3)
$$

Where $t_{i}$ is the propagation time of Lamb waves with group velocity $c_{g}$ from the $i$ th actuator/sensor to the damage. Eq.(4) can be reformulated into

$$
x^{2}+y^{2}+r^{2}-2 x x_{i}-2 y y_{i}=t_{i}^{2} c_{g}^{2}(i=1,2,3)
$$

Choosing any two equations of Eq. (5) and subtracting each other will yield another system equation

$$
x_{i j} x+y_{i j} y=T_{j i} c_{g}^{2} \quad(i, j=1,2,3, \text { and } i \neq j)
$$

Where $x_{i j}=x_{i}-y_{j}, y_{i j}=y_{i}-y_{j}, T_{j i}=t_{j}^{2}-t_{i}^{2}$. Then any two equations of the system equations of Eq. (6) can be used to solve the unknown parameters of $x$ and $y$. Here the first two equations with indices $i j=12$ and 23 are chosen to yield these two parameters

$$
\begin{aligned}
& x=\frac{y_{12} T_{32}-y_{23} T_{21}}{y_{12} x_{23}-x_{12} y_{23}} c_{g}^{2} \\
& y=\frac{y_{23} T_{21}-y_{12} T_{32}}{y_{12} x_{23}-x_{12} y_{23}} c_{g}^{2}
\end{aligned}
$$

Substitute these two parameters into the left equation of the system equation of Eq. (6) and simplify it,

$$
\left(k_{1}+k_{2}\right) c_{g}^{4}-\left(2 k_{1} x_{3}+2 k_{2} y_{3}+t_{3}^{2}\right) c_{g}^{2}+x_{3}^{2}+y_{3}^{2}=0
$$


Where $k_{1}=\frac{y_{12} T_{32}-y_{23} T_{21}}{y_{12} x_{23}-x_{12} y_{23}}, k_{2}=\frac{x_{23} T_{21}-x_{12} T_{32}}{y_{12} x_{23}-x_{12} y_{23}}$. The group velocity can be directly solved from Eq. (8). The only required parameter, $t_{i}$ can be calculated from the estimated TOFs of incident wave from the actuator to the damage and back to the sensor with the equation

$$
t_{i}+t_{j}=\operatorname{TOF}_{i j}(i, j=1,2,3, \text { and } i \neq j)
$$

After the TOFs are obtained from Eq. (8), the group velocity and the damage position can be then calculated with Eqs. (8) and (7), respectively.

The algorithm shows that the exact damage position can be determinately solved with three TOFs values, and the group velocity is theoretically computed with the estimated TOFs. Since the group velocity, which plays s straightforward role in the accuracy of damage localization, is not need to be measured additionally, thereby the potential source of localization error is reduced. When damage position is far from the sensor network, the TOFs values can be directly acquired from the damaged signal. Hence, this damage localization algorithm can be used for baseline-free SHM. In the case that damage occurs in the vicinity of the sensor network, the TOFs values need to be obtained by subtracting the baseline signal from the damaged signal. Hence it can be considered that there are no blind zones for this algorithm.

\subsection{Time-frequency analysis method-based signal processing}

As commented in the section 2, the dispersive and multimode characteristics of Lamb waves complicate the interpretation of Lamb waves. Meanwhile, various interferences such as the ambient vibration and natural structural vibration and the measurement noise are also inevitably contained in the sampled signals. For this reason, appropriate signal processing methods must be applied to the acquired raw signals.

Currently time-frequency analysis methods, especially wavelet transform (WT) are most popularly used for Lamb waves signal analysis due to its adaptive time-frequency resolution characteristics. More recently a new time-frequency analysis method called frequency slice wavelet transform (FSWT) has been developed by Yan et al. (2009). This method possesses the similar adaptive resolution properties as CWT. Furthermore, this method shows more flexibility and convenience for analyzing and processing complex non-stationary signal. More discussion and comparison of this method with other time frequency analysis methods can be found from the literature of Yan et al. (2009). Here this method is briefly stated and then applied to process the acquired raw signal.

Theoretically, the FSWT of a signal $f(t) \in L^{2}(R)$ is defined as (Yan et al. 2009)

$$
W(\omega, t)=\frac{1}{2 \pi} \int_{-\infty}^{+\infty} \hat{f}(u) p^{*}\left(\frac{u-\omega}{\sigma}\right) e^{i u t} d u
$$

Where asterisk denotes the complex conjugate, $\hat{f}(u)$ is the Fourier transform of $f(t)$ and is the frequency slice function (FSF) in frequency domain, which is similar to the wavelet basis of CWT, $\sigma$ is the scale parameter in frequency domain. Here it is assumed that the parameter $\sigma$ is proportional to the analyzing frequency, $\omega$, and taken as $\sigma=\frac{\omega}{\kappa}$. The FSWT is then expressed as

$$
W(\omega, t)=\frac{1}{2 \pi} \int_{-\infty}^{+\infty} \hat{f}(u) p^{*}\left(\kappa \frac{u-\omega}{\omega}\right) e^{i u t} d u
$$


The value of $\kappa$ can be specified based upon the characteristics of the signal to be analyzed. From the expression of FSWT it is known that the window width of FSF $\hat{p}$ is narrow at low frequencies and wide at high frequencies. Therefore, FSWT also has the adaptive resolution same as CWT. Nevertheless, the reconstruction condition for FSWT is $\hat{p}(0) \neq 0$ or $\hat{p}(0)=1$, which is obviously much weaker than the admissibility condition of CWT and hence can be satisfied more easily. As a result, many functions can be used as FSFs, such as

$$
\text { (I) } p(\omega)=e^{-\frac{1}{2} \omega^{2}} ;(\mathrm{II}) p(\omega)=\left\{\begin{array}{l}
1|\omega| \leq \omega_{0} \\
0|\omega|>\omega_{0}
\end{array} ;(\mathrm{III}) \hat{p}(\omega)=\frac{\sin \omega}{\omega} ;(\mathrm{IV}) \hat{p}(\omega)=\frac{1}{1+|\omega|^{n}}\right.
$$

The inverse FSWT to reconstruct the original signal is expressed as

$$
f(t)=\frac{1}{2 \pi} \int_{-\infty}^{+\infty} W(\omega, t) e^{i \omega(t-\tau)} d \tau d \omega
$$

Eq. (12) shows that the inverse FSWT is not directly in relation with the FSF or $\hat{p}(\omega)$, and hence the reconstruction of signal with FSWT is more convenient than that of CWT. At the same time, the inverse FSWT can be efficiently performed with fast Fourier Transform algorithm, which is another advantageous feature of FSWT over CWT.

In the present work, $\hat{p}(\omega)=e^{-\frac{1}{2} \omega^{2}}$ is adopted as FSF for signal processing, as it has the smallest Heisenberg resolution in the time-frequency plane. In order to eliminate the adverse interferences described above, a filtering process is developed in Eqs. (13)-(14), allowing de-noising inside the narrowband of the input signal

$$
\begin{gathered}
W(\omega, t)=0 . \text { for } \omega<\omega_{\text {lower }} \text { or } \omega>\omega_{\text {lower }} \\
\left\{\begin{array}{l}
W(\omega, t)=0, \text { if }|W(\omega, t)|<\theta \\
W(\omega, t)=\operatorname{sgn}(W(\omega, t)) \cdot(|W(\omega, t)-\theta|), \text { if }|W(\omega, t)| \geq \theta
\end{array} \text { for } \omega_{\text {lower }} \leq \omega<\omega_{\text {upper }}\right.
\end{gathered}
$$

Where $\omega_{\text {lower }}$ and $\omega_{\text {upper }}$ is respectively the lower and upper limit of the known excitation frequency range, $\theta$ is the chosen threshold of the coefficients of FSWT. Finally, the obtained result is converted to time domain with inverse FSWT.

\section{Experimental results}

Experiment is performed to verify the damage localization algorithm. In the experiment the damage is realized through clamping a C-clamp with contact diameter of $20 \mathrm{~mm}$ onto plate. The simulated damage changes the stiffness and the inertia of the structure in a localized area, causing the incident Lamb waves to be scattered from it, hence it has the similar effect as crack. Here this method is taken because it is easy to manipulate and allows for repeatable test of different damage configuration. Fig. 8 shows the aluminum plate with the collocated PZT sensor network and C-clamp.

The raw signal measured via the actuator-sensor pair of P1-2 before and after the occurrence of the 'damage' is displayed in Figs. 9(a) and 9(b). From comparing the signals shown in Figs. 9(a) and 9(b), the appearance of the noise in the signal obviously affects the precise determination of the TOFs 


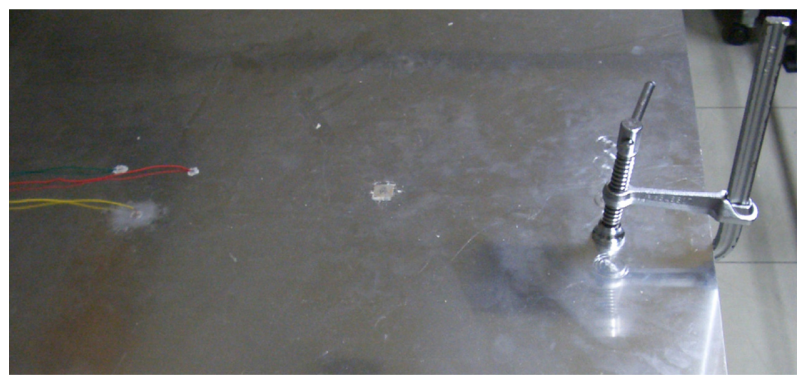

Fig. 8 Photograph of the experimental setup

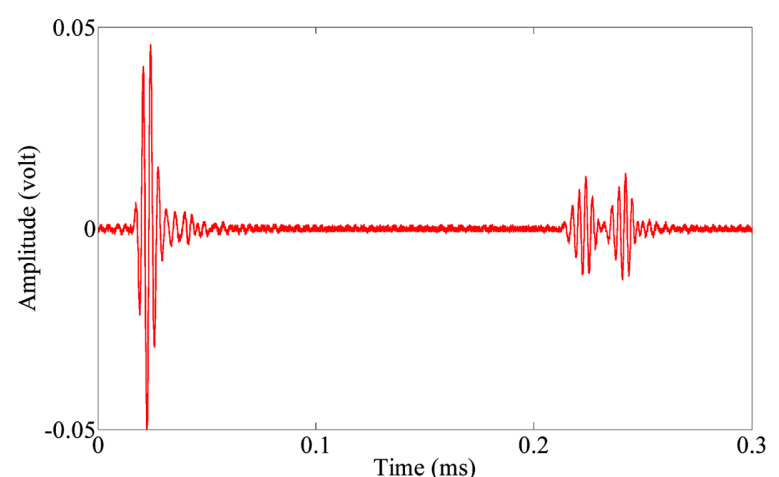

(a)

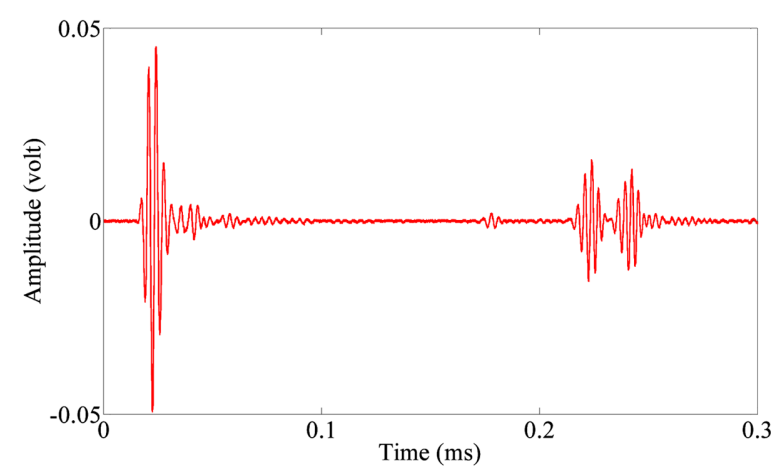

(b)

Fig. 9 Raw Lamb wave signal via P1-2 measured at (a) healthy state and (b) damaged state

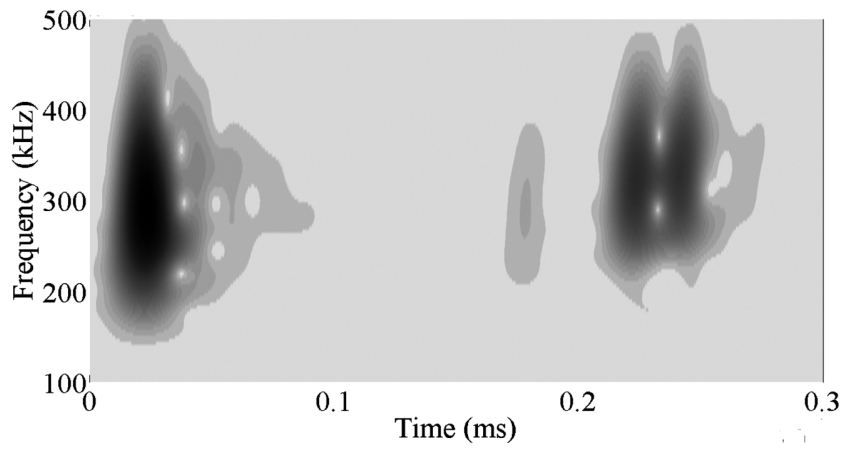

Fig. 10 Time-frequency plane of the raw signal via P1-2 at damaged state

of wave reflected from the damage. In the next, FSWT is applied to the raw signal with $\kappa=6.0$. The resulting time-frequency plane is shown in Fig. 10. The filtering process is carried out with $\theta=0.0001$ and the reconstructed signal are shown in Fig. 11, the TOF value is determined from the arrival time of the amplitude maximum of the envelope of the reconstructed signal, the envelope is obtained by summing the absolute value of the filtered FSWT coefficients over the frequency axis at each time point. The three envelopes of the reconstructed signal are shown in Figs. 12(a)-12(c), respectively.

After a set of TOFs is known from the envelopes of the signals, the position of damage can be then quickly estimated using the localization algorithm. The result of damage localization is described in 


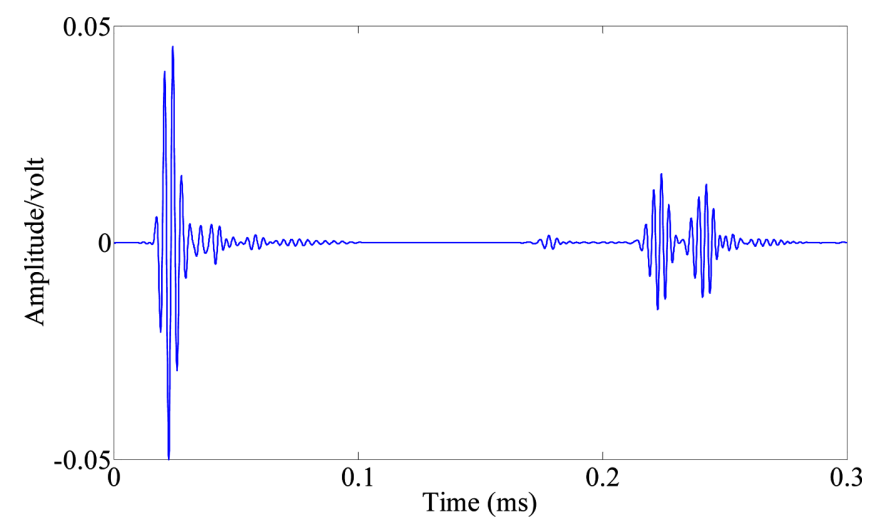

Fig. 11 Reconstructed signal via P1-2

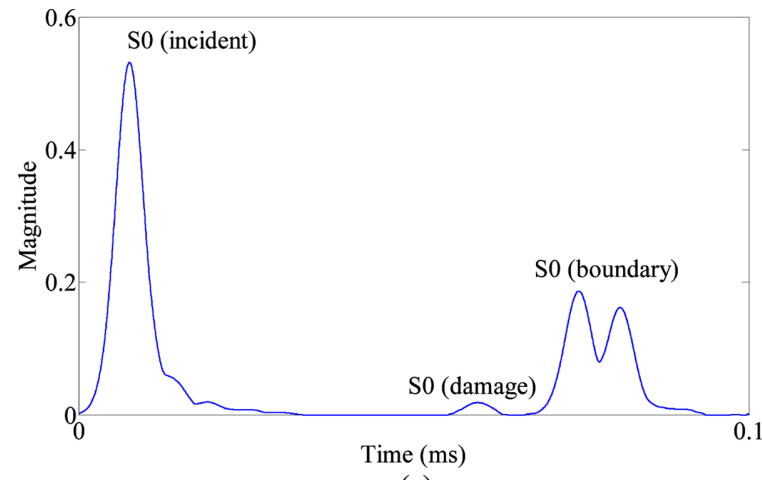

(a)

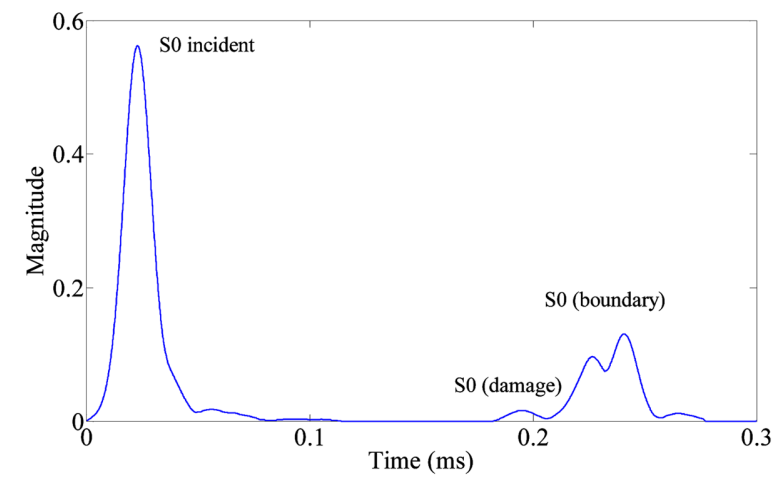

(b)

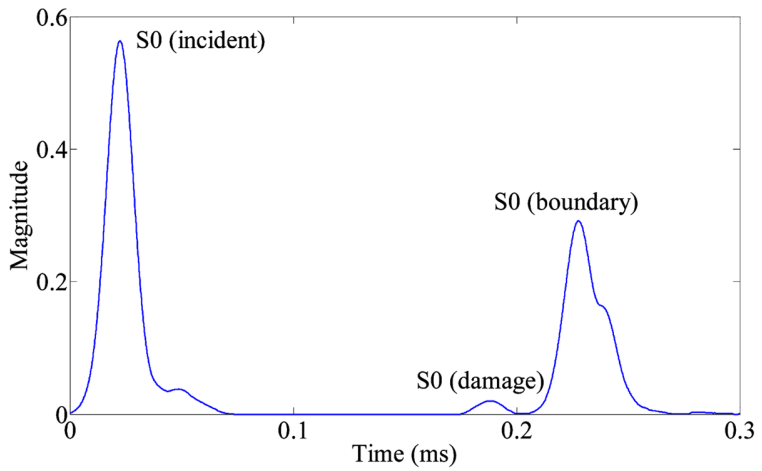

(c)

Fig. 12 The envelope of the reconstructed signal via (a) P1-2, (b) P1-3 and (c) P2-3

Table 2 and schematically illustrated in Fig. 13.

In the present case, the algorithm gained good accuracy for locating the damage within plate. However, the influence of the boundary of the structure on the application of the algorithm is addressed. Since the reflected signal from the damage is only used for the algorithm, if only the signal from the damage is not contaminated by the signal from the boundary, the accuracy of the algorithm will not be affected, there is no need for considering the boundary conditions. However, 
Table 2 Estimated result of damage position

\begin{tabular}{cccc}
\hline \hline No & Actual position $(x, y)(\mathrm{mm})$ & Estimated position $(x, y)(\mathrm{mm})$ & Error $(\%)$ \\
\hline 1 & $(495.0,0)$ & $(492.9,-12.4)$ & 0.38 \\
2 & $(495.0,100)$ & $(477.9,141.9)$ & 1.98 \\
3 & $(0,-495.0)$ & $(16.6,-506.0)$ & 2.26 \\
4 & $(495.0,-60.0)$ & $(483.1,-57.7)$ & 2.41 \\
\hline
\end{tabular}

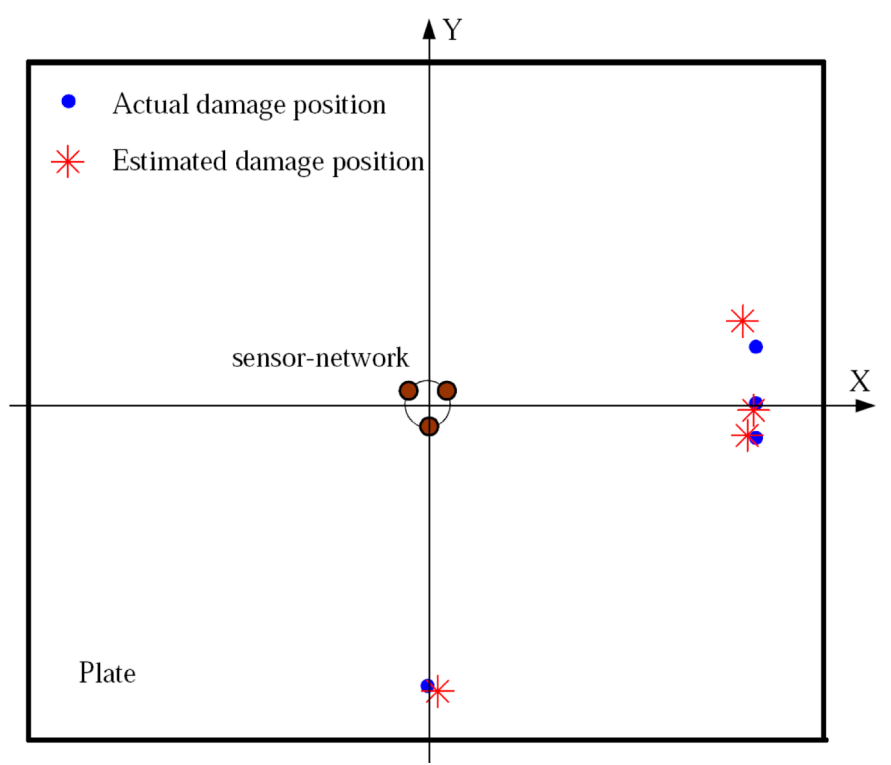

Fig. 13 Schematic damage localization results

when the damage is located close to the boundaries, the reflected signal from damage may be overlapped by the signal from boundaries, there will be some ambiguity in identifying the arrival of the reflection wave from damage, the accuracy of the algorithm will be affected adversely. In such case, the signal from the boundary must be removed from the damaged signal damage using the baseline signal.

\section{Conclusions}

A piezoelectric sensor network consisting of three PZT wafer active sensors placed on a circle configuration is proposed to locate damage in plate. The three PZT active sensors are used to alternately excite and detect Lamb waves. Based upon the tuning effect of the interaction of Lamb waves with piezoelectric active sensor, an appropriate excitation frequency is designed to generate a single $S_{0}$ mode. A localization algorithm based upon the configuration of PZT sensor network is developed. The advantages using this damage localization algorithm includes several aspects: first the exact damage position can be efficiently solved, with no existence of blind zones over the inspection areas. The group velocity is directly known from the measured TOFs, and hence reducing the potential error of damage localization. A new time-frequency analysis method FSWT is applied to 
eliminate the adverse influence of various interferences and measurement noise. The experimental result obtained from the aluminum plate demonstrates that the damage position can be accurately estimated with the approach.

\section{References}

Achenbach, J.D. (1984), Wave Propagation in Elastic Solids, North-Holland, New York.

Giurgiutiu, V., Zagrai, A. and Bao, J.J. (2002), "Piezoelectric wafer embedded active sensors for aging aircraft structural health monitoring", Struct. Health Monit., 1(1), 41-61.

Giurgiutiu, V. (2005), "Tuned Lamb wave excitation and detection with piezoelectric active sensors for structural health monitoring”, J. Intel. Mat. Syst. Str., 16(4), 291-305.

Grondel, S., Paget, C., Delebarre, C., Assaad, J. and Levin, K. (2002), "Design of optimal configuration for generating A 0 Lamb mode in a composite plates using peizoceramic transducers", J. Acoust. Soc. Am., 112, 84-90.

Hu, N., Shimomukai, T., Fukunaka, H. and Su, Z.Q. (2008), "Damage identification of metallic structure using Ao mode of Lamb waves", Struct. Health Monit., 7, 271-285.

Lin, X. and Yuan, F.G. (2002), "Diagnostic Lamb waves in an integrated piezoelectric sensor/actuator plate: analytical and experimental studies", Smart Mater. Struct., 10(5), 907-913.

Su, Z.Q., Ye, L. and Bu, X.Z. (2002), "A damage identification technique for CF/EP composite laminates using distributed piezoelectric transducers", Compos. Struct., 57(1-4), 465-471.

Tua, P.S., Quek, S.T. and Wang, Q. (2004), "Detection of cracks in plates using piezo-actuated Lamb waves", Smart Mater. Struct., 13(4), 643-660.

Viktorov, I.A. (1967), Rayleigh and Lamb waves, physical theory and applications, Plenum, New York.

Wang, C.S.F. and Chang, F.K. (2001), "Structural health monitoring from fiber-reinforced composites to steelreinforced concrete", Smart Mater. Struct., 10(3), 548-552.

Wang, L. and Yuan, F.G. (2007), "Active damage localization technique based on energy propagation of Lamb waves", Smart Struct. Syst., 3(2), 201-207.

Wieslaw, O, Pawel, K., Pawel, M. and Tomasz, W. (2009), "Damage localization in plate-like structures based on PZT sensors", Mech. Syst. Signal Pr., 23(6), 1805-1829.

Yan, Z.H., Miyamoto, A. and Jiang, Z.W. (2009), "Frequency slice wavelet transform for transient vibration response analysis", Mech. Syst. Signal Pr., 23(5), 1474-1489.

$C C$ 